\title{
Positive correlation between TZAP and TERT in most cancers: a new player in cancer diseases
}

\author{
Yu-Ran Heo, Won-Jin Park, Jae-Ho Lee \\ Department of Anatomy, Keimyung University School of Medicine, Daegu, Korea \\ Correspondence to: Jae-Ho Lee. Department of Anatomy, Keimyung University School of Medicine, 2800 Dalgubeoldaero, Dalseo-Gu, Daegu, Korea. \\ Email: anato82@dsmc.or.kr. \\ Response to: Donati B, Valenti L. Telomeric zinc-finger associated protein (TZAP): a new player in telomere diseases? Ann Transl Med 2017;5:472.
}

Submitted Feb 08, 2018. Accepted for publication Mar 07, 2018.

doi: $10.21037 / \mathrm{atm} .2018 .03 .14$

View this article at: http://dx.doi.org/10.21037/atm.2018.03.14

We read with great interest the article published in this journal by Donati et al. (1), which suggested that telomeric zinc-finger associated protein (TZAP) dynamics may lead to the development of telomere diseases. According to the Human Protein Atlas, TZAP (also known as ZBTB48) is expressed in various cancers, and its role as a tumorsuppressor is suspected $(2,3)$.

In January 2018, TZAP mRNA expression data for various cancers were downloaded from The Cancer Genome Atlas (TCGA) database (http://cancergenome. nih.gov/) (3). We used OncoLnc and cBioPortal to analyze the expression of TZAP and found that TZAP expression had prognostic significance in colorectal, cervical, and pancreatic cancers. In other cancers, it did not have any prognostic value. In addition, TZAP expression positively correlated with telomerase reverse transcriptase (TERT) expression in bladder, colorectal, lung, head, neck, and uterine endometrial squamous cell carcinomas, as well as hepatocellular carcinoma, glioblastoma, and melanoma (Figure 1). Those correlations had not been reported until now.

The shelterin subunits telomeric repeat factor (TRF) 1 and TRF2 $(2,4)$ are known to compete with TZAP. In our study, TZAP expression strongly correlated with TERT expression, and the mRNA of each was competitively and proportionally expressed. Thus, it was believed that the competition between TZAP and the shelterin complex may be limited to certain locations. Moreover, TZAP expression may be more deeply associated with TERT expression, as suggested by Donati et al. (1). Further research into how TZAP expression and binding affect the molecular mechanisms of telomere crosstalk with other cellular processes is warranted.

Our analysis, combined with previous data, suggests that TZAP directly participates in telomere regulation in a manner that is dependent on TERT $(1,2)$, which indicates that TZAP expression may contribute to the pathogenesis of telomere-related diseases such as cancer. For that reason, the biological functions of TZAP and telomereassociated gene alterations are of great interest to cancer researchers.

\section{Acknowledgements}

Funding: This study was supported by grants from the Basic Science Research Program through the National Research Foundation of Korea (NRF), which is funded by the Ministry of Education (NRF-2014R1A6A3A04058057). 


\begin{tabular}{lcc} 
A & & \\
\hline Cancer type & $\mathrm{R}$ & $\mathrm{P}$ \\
\hline Bladder urothrlial & 0.251 & $0.000^{*}$ \\
Breast invasive carcinoma & 0.058 & 0.065 \\
Cervical squamous cell carcinoma and & -0.014 & 0.091 \\
Colon adenocarcinoma & 0.187 & $0.000^{*}$ \\
Esophageal carcinoma & 0.077 & 0.359 \\
Glioblastoma multiforme & 0.232 & $0.004^{*}$ \\
Head and neck squamous cell carcinoma and & 0.216 & $0.000^{*}$ \\
endocervical adenocarcinoma & & \\
Acute myeloid leukemia & 0.118 & 0.089 \\
Liver hepatocellular carcinoma & 0.177 & $0.001^{*}$ \\
Lung adenocarcinoma & 0.065 & 0.152 \\
Lung squamous cell carcinoma & 0.115 & $0.011^{*}$ \\
Ovarian serous cystadenocarcinoma & 0.003 & 0.963 \\
Pancreatic adenocarcinoma & -0.018 & 0.810 \\
Rectum adenocarcinoma & 0.186 & $0.020^{*}$ \\
Skin cutaneous melanoma & 0.134 & $0.004^{*}$ \\
Stomach adenocarcinoma & 0.088 & 0.089 \\
Uterine corpus endometrial carcinoma & 0.133 & $0.002^{*}$ \\
\hline
\end{tabular}
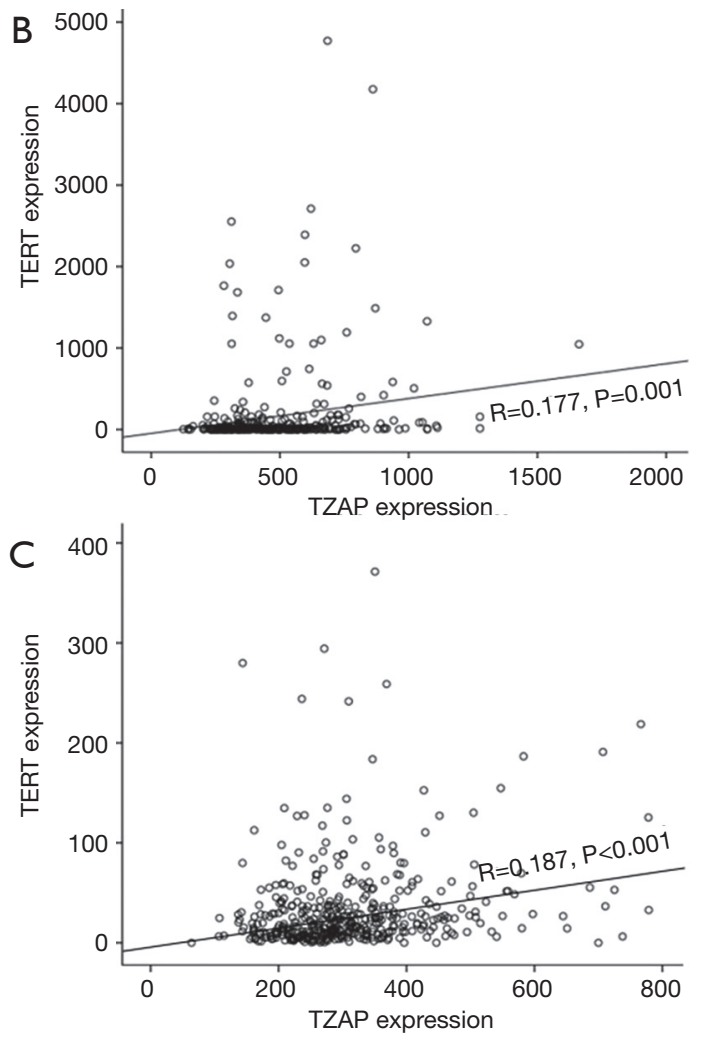

Figure 1 Correlation between TZAP and TERT expression in various cancers (A), representative correlation data on hepatocellular carcinomas (B) and colon cancers $(\mathrm{C})$. *, $\mathrm{P}<0.05$. TZAP, telomeric zinc-finger associated protein; TERT, telomerase reverse transcriptase.

\section{Footnote}

Conflicts of Interest: The authors have no conflicts of interest to declare.

\section{References}

1. Donati B, Valenti L. Telomeric zinc-finger associated protein (TZAP): a new player in telomere diseases? Ann Transl Med 2017;5:472.

Cite this article as: Heo YR, Park WJ, Lee JH. Positive correlation between TZAP and TERT in most cancers: a new player in cancer diseases. Ann Transl Med 2018;6(10):197. doi: 10.21037/atm.2018.03.14
2. Li JS, Miralles Fusté J, Simavorian T, et al. TZAP: A telomere-associated protein involved in telomere length control. Science 2017;355:638-41.

3. Anaya J. OncoLnc: linking TCGA survival data to mRNAs, miRNAs, and lncRNAs. Peer J Computer Science 2016;2:e67.

4. Pickett HA, Cesare AJ, Johnston RL, et al. Control of telomere length by a trimming mechanism that involves generation of t-circles. EMBO J 2009;28:799-809. 